\title{
Energy flexibility of office buildings - Potential of different building types
}

\author{
Mingzhe Liu ${ }^{1, *}$, Hicham Johra ${ }^{1}$, Per Kvols Heiselberg ${ }^{1}$, Ivan Kolev $^{1}$, Kremena Pavlova $^{1}$ \\ ${ }^{1}$ Department of Civil Engineering, Thomas Manns Vej 23, 9220 Aalborg Ø, Denmark
}

\begin{abstract}
The objective of this study is to investigate and assess the energy flexibility performance of typical Danish office buildings constructed at different periods. Four building study cases have been compared with different heating demands, structural thermal masses, envelope insulation levels and infiltration rates. All cases are equipped with the same novel two-pipe heating and cooling system. Each case is divided in four subcases with variations of heat gains: people load, lighting load, equipment load, solar gain. Analyses and comparisons have been performed on different parameters, including power load shifting and grid adjustment, comfort level, and economical benefits. All investigated cases are tested with two control strategies: a normal reference control strategy and an energy flexibility control. The flexible controller adjusts the indoor temperature set points for heating and cooling depending on different energy price levels.
\end{abstract}

\section{Introduction}

Energy flexibility is a growing activity of research. Buildings can store energy with equipment like water tanks and batteries, or the building structure itself [1-3]. In this way, they can adjust their energy demand, which can help in solving the problems with future energy grids dominated by intermittent renewable energy sources.

There is a promising potential of the thermal mass of the building structure. Therefore, in different case studies, it is commonly evaluated how thermal structure of a building can take best advantage of fluctuating energy prices [1-3]

As stated in [3], the investigation approach of such cases confirms for high energy cost savings when thermal mass is being activated in order to achieve optimized energy consumption profile. However, it is also mentioned that the results are highly case dependent, due to the fact the savings vary of the local energy price market and thus can be difficult to generalize conclusions. Electricity production provided by renewable sources has relatively unpredictable trend. This surplus or lacks of power in grid influence the price on market. Buildings can benefit from the price fluctuation if they make their energy systems flexible and oriented to low tariff periods. Application of energy flexibility will also make them supportive to the challenges in electricity grid.

There are constructions currently used as office buildings in Denmark, which are erected over different ages. Some are quite modern, others have typical features from the middle of $20^{\text {th }}$ century, there are even architectural examples dating back to the "National Romanticism" period. All these variety of building types will affect the performance of energy flexibility differently. Therefore, the focus of this paper and the main research question is: What is the energy flexibility potential in office buildings from different periods of time? The approach to the question would be set by distinguishing different building properties typical for each identified period. Once the classification of the constructions and their parameters are clarified, their energy flexibility performance will be simulated using a relevant software tool (EnergyPlus). Then performance of each type will be examined and compared according to evaluation metrics.

\section{Methodology}

Focuses of investigations are office buildings which have typical properties for four different periods of timeadministrative buildings from 1890-1930, from 19401980, complying "Building regulations 2015", and fulfilling "Building Class 2020". Periods are distinguished in 4 different cases which differ in transmission and infiltration losses, and heat capacity of building elements. The 4 cases are combined with 2 alternatives for people load, equipment load, lighting load, and another 2 alternatives for g-values. Therefore, all cases have 4 variations resulting in total of 16 different options. All options are being tested for their energy performance with and without a flexibility controller of their HVAC system. EnergyPlus is being used to perform the simulations, as it has the ability and functionality to implement the specific heating and cooling system together with the used control strategy. The building model used in simulations has the same geometrics for all cases. It is a three-storey building with

Corresponding author: ml@,civil.aau.dk 
total area of $2926 \mathrm{~m}^{2}$. The model has a relatively high share of $38 \%$ glazed facade area. The pitched roof typical for case 1 is simplified and shape is adjusted to fit the software model. Weather data file used in simulations complies for Denmark (Copenhagen). Energy flexibility of all options is tested with an archived electricity price list from 2015 [1]. The results of all investigated options are evaluated and compared according to evaluation metrics.

\subsection{Building Case studies}

Currently in Denmark, there are buildings used for office and administrative purposes constructed in different periods of time. Some of them are quite newly executed, but also there are buildings which time of original erection can be tracked back to the end of $19^{\text {th }}$ century. Each period specifies with its own constructing properties like air tightness, level of insulation, material use, thermal mass, etc. As those properties differ for each period, a comparison is done among them in order to investigate their response to energy flexibility, and more specifically the potential to conserve energy. In this research four main building periods are distinguished, which can be seen in the form of four cases (figure 1).

\subsection{HVAC system and Control strategies}

The case study involves evaluation of the energy flexibility potential of a novel building energy system that is represented by active chilled beam used for both heating and cooling [5].

Such system is based on induction principle and the heat transfer is done by forced convection. Ventilation air is supplied with a high pressure by the diffusers of the beams located on the suspended ceiling of each thermal zone. The created low pressure underneath the unit makes the room air to induce through the coil and then this air is recirculated again through the diffuser and supplied back in the room heated or cooled, depending on the need. Ventilation ratio of primary to induction air can vary between $1: 2$ and 1:7, which is quite high for an active chill beam. The primary air is supplied in the unit with a set point of 18 degrees and for this purpose is preheated by the use of a rotary heat exchanger.

The unique HVAC system allows low temperature heating and high temperature cooling handled by the same two-pipe water loop with operating temperatures of 20-23 degrees.

This will provide the possibility of reusing the thermal energy in the return water circuit, which makes the system highly efficient and will contribute to greater savings on running and commissioning costs. As well as cost for repair of the system, due to the fact there are only pipes for maintenance (e.g. there are no regulation valves).

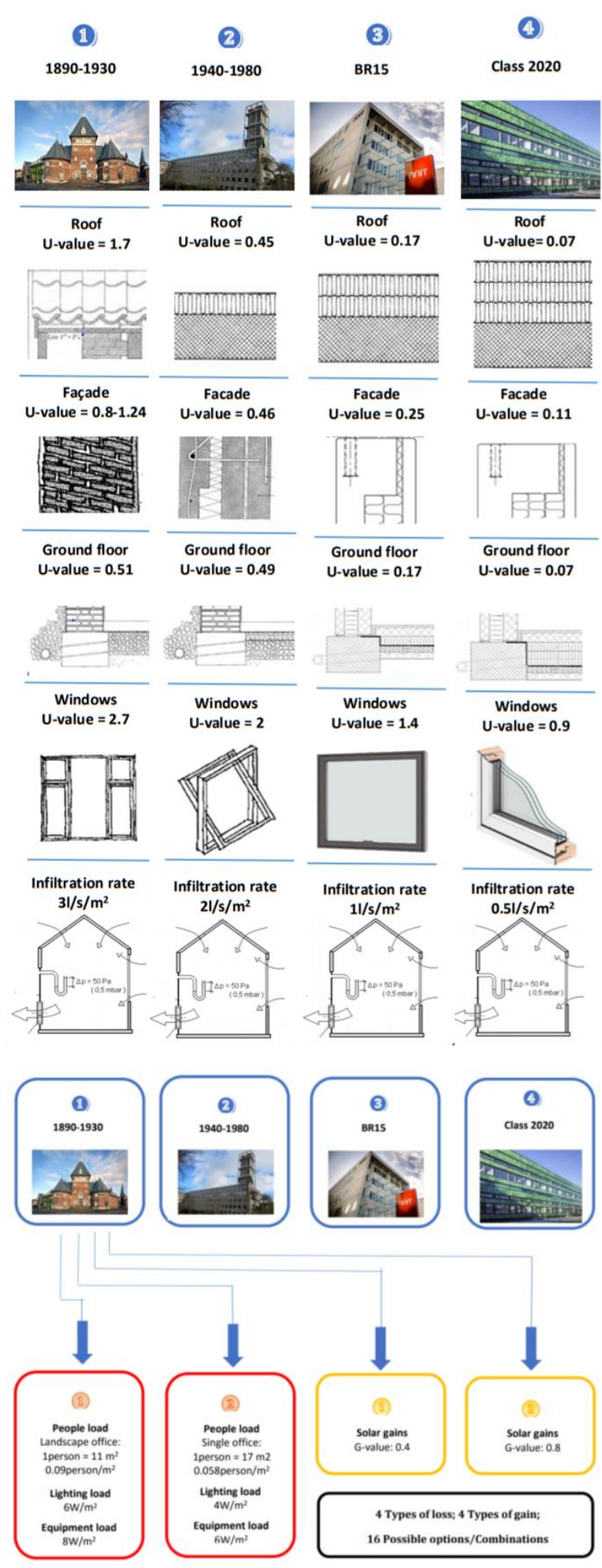

Fig. 1. Different building types.

The innovative two pipes system will also provide the possibility to cool and heat in the same time the building as the system can take an advantage from the excess heat from one place and transfer it to another if there is a need for this. 


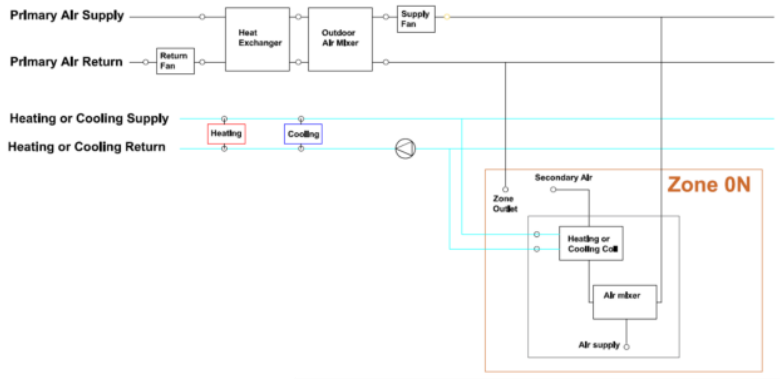

Fig. 2. Energy system in the case building.

Two control strategies are carried out in order to research the energy flexibility potential of the studied building cases. The first one is a normal controller scenario, which operates the HVAC system under normal conditions by only following two set points -one for occupied and another for unoccupied hours both for heating and cooling. The second control - a flexibility controller, based on more complex control algorithms provides different set points for heating and cooling depending on the electricity price. This control works by adjusting the set points of the building system to three electricity price levels low, medium and high, which thresholds are derived from electricity price data for 2015 [1].The concept of the flexibility controller is to either increase or decrease the set points during low price electricity for heating or cooling and in this way to store energy in the thermal mass of the construction. It is expected this action will provide good thermal comfort when high price electricity is available and even though in this period the system will perform on lower power mode.

\section{Evaluation metrics}

The evaluation metrics are defined considering the benefits of the grid, building owners and building users. It includes the ability of power adjusting, the coefficient of energy shifting, economic benefit and comfort level. By simulating the specific office building, the metrics are calculated and compared to evaluate both yearly and seasonal performance of the building under different control strategies.

\subsection{Ability of power adjustment}

As one of the most important parameters to the grid, this metric defines the ability of a building to adjust power during high price periods and low price periods, which is also used by the authors in [2]. Buildings are expected to reduce more power demand during high-price periods and thereby contribute to the reduction of the peak load of the grid. Additionally, the amount of power that the building increases during low-price is still important, especially when more and more buildings are capable of controlling the energy system according to price. The metric is presented as hourly power difference between a case with control of demand response and a reference case without control of demand response $\left(\mathrm{P}_{\text {difference }}\right)$, calculated by equation (1). Where $\mathrm{P}_{\text {flexibility }}$ and
Preference are the hourly power supplied to the building with the energy flexibility control and to the reference case, respectively.

$$
P_{\text {difference }}=P_{\text {flexibility }}-P_{\text {reference }}
$$

\subsection{Energy flexibility factor}

In order to activate the thermal mass of building construction elements, the control strategies of demand response need to supply extra heating or cooling energy to the building when the price of electricity is low. The energy is expected to be stored in the thermal mass and released passively later when it is needed during highprice periods. The metric has been used by other researchers to evaluate the performance of energy flexibility for different buildings with different control strategies [29].

$$
F_{\text {Flexibility }}=\frac{\int_{L \text { w }} q_{\text {heating+cooling }} d t-\int_{\text {High }} q_{\text {heating+cooling }} d t}{\int_{\text {Low }} q_{\text {heating }+ \text { cooling }} d t+\int_{\text {High }} q_{\text {heating+cooling }} d t}
$$

This metric is presented as the factor of energy flexibility $\left(\mathrm{F}_{\text {Flexibility }}\right)$ calculated by equation (2). Where $\mathrm{q}_{\text {heating+cooling }}$ is the hourly energy consumption of heating and cooling. is the sum of all the periods when the price is low and is the sum of all the periods when the price is high. dt is the time step, which is one hour in the simulations in this study. According to the equation, the flexibility factor is from -1 to 1 . The energy flexibility of around 0 was calculated in [3] for a reference case.

\subsection{Economic benefit}

Another important metric for owners or tenants is the energy cost of a building, which has been used and suggested by other researchers. By regulating building energy systems during a period based on energy price, it is expected that the owners or tenants will gain economic benefits on the energy bill. It is evaluated as an accumulated value (yearly energy cost [EUR/m2.per.year]) based on the hourly power demand. The yearly energy cost for heating and cooling (C) is calculated by equation (3) according to the price scheme in Denmark. Where Q is the hourly energy consumption in $\left[\mathrm{kWh} / \mathrm{m} 2\right.$.per.hour]; $\mathrm{P}_{\mathrm{EL}}$ is the electricity price which is changing during the year in $[\mathrm{EUR} / \mathrm{kWh}] ; \mathrm{P}_{\text {transmissiontax }}$ is the price for transmission and tax in [EUR/kWh], which is almost constant all the time during the year. It is important to note that the transmission and tax costs $\left(\mathrm{P}_{\text {transmission }+ \text { tax }}\right)$ take around $83 \%$ of the total cost of electricity in Denmark (2018) with the value of 0.224 [EUR/kWh] and does not variate during the year, which makes it difficult for the users to benefit economically even with a very optimal control strategy. More results and discussions are shown in the result section.

$C=\int\left(Q \cdot P_{E L}\right) d t$

\subsection{Comfort level}


One common strategy of providing demand response control using the thermal mass of a building construction is to adjust heating and cooling set-points, which leads to the possibility that the indoor thermal comfort will be influenced. Comparison of comfort level needs to be conducted in order to be able to evaluate if the demand response control will sacrifice indoor thermal comfort. Requirements from EN 15251 [6] are used in the study to compare the influence of different strategies on indoor environment.

\section{Results}

According to the performed yearly dynamic simulations and analyses over the different building typologies, it can be generally concluded that the highest energy flexibility is accomplished in the buildings with high thermal performance- Case 3 and 4. The better thermal structure has greater ability to provide necessary conditions for energy flexibility. Additionally, the thermal comfort is preserved, and even slightly improved in the building class 2020-Case 4, particularly for comfort Class I. The savings from the annual energy costs are varying for each option of the mentioned cases. However, it is investigated a significant increase of savings on energy cost per $\mathrm{m} 2$ per year in opt $13-41 \%$. Consequently, high ability of energy shifting from high to low price electricity is observed in the same cases with evidently high flexibility coefficients.

As for the buildings with poorest thermal performance Case 1, flexibility controller did not perform as expected. The flexibility approach here is considered as inadequate for a building with such thermal characteristics. The yearly energy consumption is highly influenced and the cost for electricity jumped with 13 to $16 \%$ in comparison with the reference case. There are also low flexibility factors, which suggest for lower efficiency of this controller.

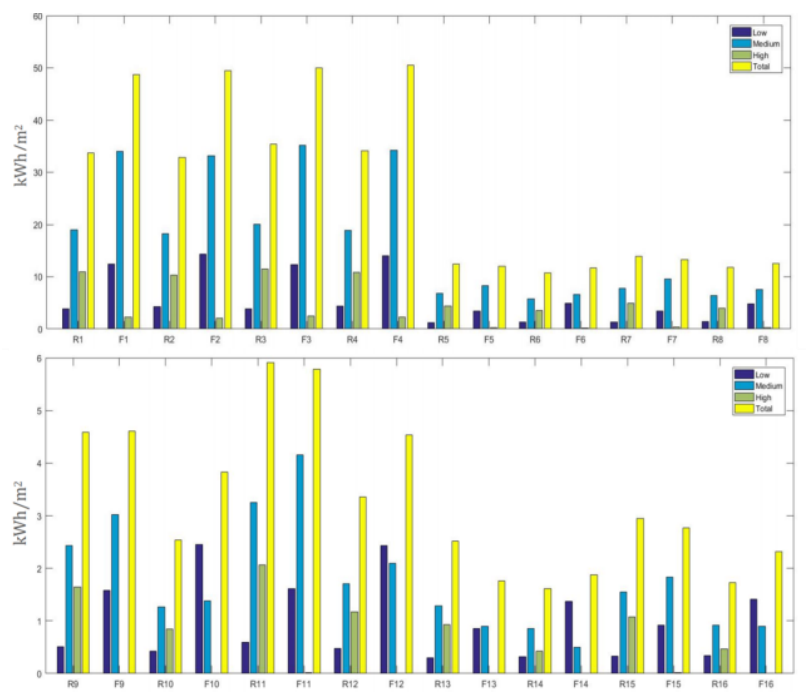

Fig. 3. Comparison of energy consumption between reference and flexibility for all options
In building Case 2, the thermal comfort is decreased by approximately $3 \%$. On the other hand, this control application can bring high savings of up to $26 \%$ from the yearly electricity consumption costs per m2. After all, insignificant differences in comfort can be neglected if the running costs of the building system are prioritized. This case demonstrates fair energy flexibility factors, but only in options with higher internal gains.

Figure 3 verifies the perceived tendency of energy shifting that the flexibility controller tends to work efficiently from building case 2 (Option 5-8). It is registered that approximately 80 to $90 \%$ of high price energy consumption is decreased in flexibility cases compared to reference ones. While the low and mainly medium price energy usage is increased in flexibility options, but still resulting in savings of el price per $\mathrm{kWh} / \mathrm{m} 2$ per year compared to references.

As for the buildings with lower heating demand (case 3 and 4), the high price energy usage is completely excluded from the flexibility options as shown on figure 3. The higher thermal performance of these building options is due to increased level of insulation and reduced infiltration rate. It is noticeable, the energy shifting potential is at its optimum in options 10 and 14, respectively building types 3 and 4 (high solar and internal gains). These options demonstrates no electricity consumption at high price levels, high energy usage during low price periods and minimized as possible medium price electricity utilization as seen on figure 3 .

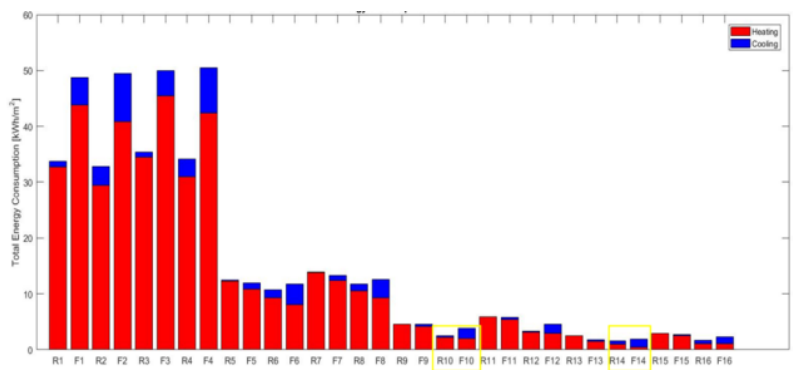

Fig. 4. Annual energy consumption for heating and cooling for all building options

Figure 4 shows the results for total hourly consumption for both heating and cooling on yearly base. All assessed options are presented with a normal controller and a flexible one. Options from 1 to 4 represent building type from Case 1. Those options register significantly more consumption than all the rest. Reasons can be found in the high transmission and infiltration losses typical for the case. Furthermore, all flexibility options consume more energy than their relative reference options. Apparently higher modulation set points are achieved quite challenging. Buildings from 1890-1930 have very poor ability to trap generated heat. Storing energy in thermal mass by increased set point comes together with a lot of heat waste. Also, it is visible the high influence of solar gains. Options 2 and 4 have windows with higher g-value. This reflects to big amount of energy spent on heating, and more on cooling over the year. Case 2 sums up the options from 5 to 8 . Here the difference in consumption between reference and 
flexibility options are much smaller, but still noticeable. As the trend for this case shows that in options 5 and 7 , which have lower solar gains, the reference uses higher amount of energy yearly. And for options 6 and 8, the higher energy use annually is for the flexibility controller. The situation in case 3 and 4 is quite similar, as the trend for rest of options is the same. The difference in cases 3 and 4 is the advantage of the higher building class. The better thermal properties of the building components in case 4 results in smaller amount of energy spent on heating and cooling on annual base.

In general, lower coefficients are observed in old construction options in flexibility cases. However, the flexibility factors are becoming bigger in building case 2 , but evidently higher in building case 3 and 4 . The latter confirms for better utilization of thermal mass due to improved building envelope in the optimized construction cases. This contributes to relatively low total electricity consumption and ability of decreasing and even eliminating high price energy consumption.

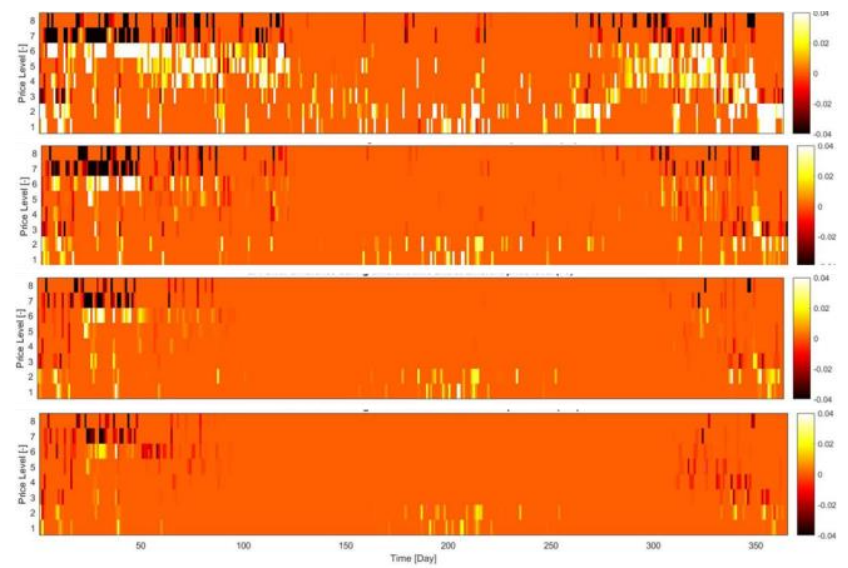

Fig. 5. Different building types (from top to bottom: option 1, 5,9 and 13).

Figure 5 shows the power difference between flexibility and reference for cases 1, 5, 9, and 13. Common for these options is they have the same people load, lighting load, equipment load, and solar gains but they all belong to different cases.

\section{Conclusions}

The study evaluates the energy flexibility potential of typical office buildings with various construction options. The building options are tested with a novel building energy system combined with flexibility and normal control strategies. The assessment is carried out by the use of several evaluation metrics: thermal comfort, economic benefit, ability of power shifting and adjustment to the grid.

Suggested constructions are defined by different thermal properties such as level of insulation, airtightness, thermal mass, etc. In total, there are established 16 options derived from 4 different building cases constructed in different periods of time. The wide range of observed models is an attempt to cover the variety of non-renovated and recently constructed buildings in Denmark.

A two pipe heating and cooling system with a flexibility control strategy based on electricity prices is profitable mainly for higher energy building classes (Buildings complying BR15 and Class 2020). Such buildings in a combination with high internal gains, which is normally the case for office buildings (high internal load from equipment, people and lighting) can contribute for relatively high savings in energy cost for heating and cooling. High solar gains influence negatively the sensitive structures from Cases 3 and 4. This leads to increased energy consumption spent on cooling, which reduces the economic benefits. Results on applied flexibility strategies for Case 2 are not satisfying, despite there are some optimistic achievements. Levels of investigated energy flexibility potential in non-renovated buildings typical for Case 1 are evaluated not just low, but even much worse compared to reference conditions.

\section{References}

1. Johra H, Heiselberg PK, Le Dréau J, Energy and Buildings 183 325-339, (2019)

2. M. Liu, P. Heiselberg, Applied Energy. 233-234, (2019)

3. Le Dreau J, Heiselberg P. Energy. 111:991-1002, (2016)

4. Reynders G, Diriken J, Saelens D. Appl Energy. 198:192-202. (2017)

5. Maccarini A, Wetter M, Afshari A, Hultmark G, Bergsøe NC, Vorre A. Energy Build. 134:234-47, (2017)

6. CEN E. 15251, Indoor environmental input parameters for design and assessment of energy performance of buildings addressing indoor air quality, thermal environment, lighting and acoustics. European Committee for Standardization, Brussels, Belgium 2007. 